\title{
A multi-center retrospective analysis of the effect of DPP4 inhibitors on progression-free survival in advanced airway and colorectal cancers
}

\author{
AZKA ALI $^{1 *}$, ALEJANDRA FUENTES $^{2 *}$, WILLIAM PAUL SKELTON IV ${ }^{1 *}$, YU WANG $^{3}$, \\ SUSAN McGORRAY ${ }^{3}$, CHINTAN SHAH $^{1}$, ROHIT BISHNOI ${ }^{1}$, LONG H. DANG $^{4}$ and NAM H. DANG ${ }^{4}$ \\ ${ }^{1}$ Department of Medicine, University of Florida, Gainesville, FL 32608; ${ }^{2}$ Department of Medicine, \\ Section of Hematology and Oncology, Louisiana State University, New Orleans, LA 70112; ${ }^{3}$ Department of Biostatistics; \\ ${ }^{4}$ Department of Medicine, Division of Hematology and Oncology, University of Florida, Gainesville, FL 32608, USA
}

Received April 12, 2018; Accepted October 2, 2018

DOI: $10.3892 / \mathrm{mco} .2018 .1766$

\begin{abstract}
Cluster of differentiation 26 (CD26), also known as dipeptidyl peptidase IV (DPP4), is a cell surface protein with exopeptidase activity and is expressed by most cell types. CD26/DPP4 is a multifunctional molecule with diverse biological effects, including regulatory effects on tumor growth, invasion and metastasis, and is a potential novel therapeutic target for selected cancers. In this study, we retrospectively analyzed diabetic patients with concurrent advanced airway or colorectal cancer to examine the effect of DPP4-inhibitors on progression-free survival (PFS). We performed a multi-center retrospective review of patients with advanced colorectal or airway (lung, head and neck) cancer and a concurrent diagnosis of diabetes. The control group included patients on metformin and a sulfonylurea, and the study group included patients on metformin and a DPP4 inhibitor. Ninety-six patients were eligible for the study. The cancers progressed in $23.7 \%$ of patients treated with DPP4 inhibitors compared to $50.9 \%$ of patients in the control group with an odds ratio of 0.303 [95\% confidence interval (CI): 0.106-0.809] and $\mathrm{P}=0.010$. There was a statistically significant improvement in PFS in the study group as compared to the control group, hazard ratio $=0.42(95 \% \mathrm{CI}$ : 0.21-0.84) and $\mathrm{P}=0.014$. There was a trend toward improvement in overall survival, although this effect was not statistically significant $(\mathrm{P}=0.11)$. Exposure to DPP4 inhibitors in the study
\end{abstract}

Correspondence to: Dr Long H. Dang or Dr Nam H. Dang, Department of Medicine, Division of Hematology and Oncology, University of Florida, 1600 SW Archer Road, Gainesville, FL 32608, USA

E-mail: long.dang@medicine.ufl.edu

E-mail: nam.dang@medicine.ufl.edu

* Contributed equally

Key words: dipeptidyl peptidase IV, cluster of differentiation, airway, colorecctal group led to higher PFS in patients with advanced colorectal and airway cancers. Additional investigations with larger patient cohorts are needed to validate the relationship between DPP4 inhibition and the clinical outcome of selected malignancies.

\section{Introduction}

Cluster of differentiation 26 (CD26), otherwise known as dipeptidyl peptidase IV (DPP4), is a multifunctional cell surface protein that is expressed by many normal cell types, with variable expression in different cancers. The diverse functions of CD26/DPP4 include its peptidase activity, through which it exerts its biological functions by regulating chemokine processing, signal transduction and glucose metabolism (1-3). CD26/DPP4 also carries out its biological functions through its association with various proteins and its interaction with the cell microenvironment (1). In some cancers, decreased CD26/DPP4 expression has been associated with cancer progression, thereby suggesting that it is a tumor suppressor; whereas, in other cancers, increased CD26/DPP4 expression has been associated with increased tumor metastasis $(1,3)$. In view of its role in cancer biology, CD26/DPP4 has been the subject of multiple pre-clinical studies suggesting that it may be a novel therapeutic target for selected tumors (1,3-6).

In addition to its role in cancer, CD26/DPP4 has an important function in glucose metabolism by enzymatically degrading the insulin-secreting hormones glucagon-like peptide-1 (GLP-1) and GLP-2 to suppress glucagon secretion. Because of this unique role, DPP4 inhibitors (e.g., sitagliptin) are currently widely used in the treatment of diabetes mellitus.

While studies with animal models have demonstrated that colorectal and lung cancers can be treated with DPP4 inhibition $(7,8)$, no published work has examined and evaluated the effect of treatment with DPP4 inhibitors on the survival of patients with advanced malignancies. In this study, we evaluated the clinical outcome of diabetic patients on DPP4 inhibitors with advanced colorectal or airway (lung, head and neck) malignancies. These particular neoplasms were selected as existing pre-clinical data suggested that CD26/DPP4 is a potential therapeutic target in these cancers, and as colorectal 
and airway malignancies have the highest prevalence, the resulting sample size was adequate for the study. Head and neck cancers were combined with lung cancer in one category (the airway group) due to similar risk factors for the cancer types. Meanwhile, late-stage malignancies were chosen to minimize the necessary follow-up time, with the primary endpoint being progression free survival (PFS). We hypothesized that patients with advanced colorectal and airway cancers treated with DPP4 inhibitors would have an improved PFS compared to patients treated with other oral hypoglycemic agents. Subgroup analysis for PFS was performed based on the type of cancer, the stage of cancer and the history of surgical resection. The overall survival (OS) of the control and study groups was a secondary endpoint.

\section{Patients and methods}

Study design. We conducted a multi-center retrospective review of patients treated with DPP4 inhibitors for diabetes with concurrent advanced colorectal and airway cancers. Retrospective chart reviews were completed at two institutions: University of Florida Health/Shands Network in Gainesville, Florida and Ochsner Health System in New Orleans, Louisiana. Institutional Review Board (IRB) approval was obtained at each institution, which included a waiver of individual patient consent. The study lasted from October 2006 (when the FDA approved the use of DPP4 inhibitors) to September 2017.

The inclusion criteria in the study group included the presence of advanced (defined as stage 3 or 4) colorectal adenocarcinoma, lung carcinoma (squamous, adenocarcinoma, or small cell) or head and neck squamous cell carcinoma, as well as a diagnosis of diabetes that was being treated with active anti-hyperglycemic therapy. The study group included patients on metformin (500-2,000 mg total daily dose) and DPP4 inhibitor agents (50-100 mg sitagliptin daily, $5 \mathrm{mg}$ saxagliptin daily or $5 \mathrm{mg}$ linagliptin daily). The control group included patients on metformin (500-2,000 mg daily) and sulfonylurea agents (2.5-10 mg glipizide daily, 5-10 mg glyburide daily or 1-4 mg glimepiride daily).

Exclusion criteria included patients with early stage cancers (stage 1 and 2), neuroendocrine pathology, less than a 1-month overlap between duration of anti-hyperglycemic therapy and the presence of advanced stage malignancy or patients on other anti-hyperglycemic therapies including insulin.

Patient selection. With assistance from the UF Integrated Data Repository (IDR) and the Ochsner Electronic Research Submission System (ERSA), patient selection was achieved by screening for ICD-9/ICD-10 codes and medication names in the aforementioned inclusion criteria. Each patient chart from the list generated by IDR/ERSA was then individually reviewed via an electronic medical record in order to omit patients who met any of the aforementioned exclusion criteria. Patient demographics and details of their diabetes (including antihyperglycemic medications and the result of the hemoglobin A1c test at the time of cancer diagnosis), cancer diagnosis (location, histologic subtype and stage), cancer treatment (chemotherapy, surgery and/or radiation), response and/or progression of cancer, and overall survival were obtained, as noted in the Results section below.
Data analysis. All data generated or analyzed during this study are included in this published article. Univariate analysis of the association between cancer progression (progressed or not progressed) and the treatment received was performed using Fisher's exact test. Then the odds ratio and 95\% confidence interval (CI) were calculated. The hazard ratios (HR) of the study and control groups and their 95\% CIs were estimated from univariate or multivariate Cox Proportional Hazard models with PFS as the outcome. Survival curves of PFS and OS were generated using the Kaplan-Meier test. PFS was defined as time in months from diagnosis until clinical or radiological progression of cancer. OS was defined as time in months from diagnosis until death. The log-rank test was used to compare the survival curves between the study and control groups. All data analysis was conducted with SAS 9.4 by the biostatisticians in the Department of Biostatistics at UF.

\section{Results}

Demographics. Initially, IDR/ERSA generated a combined list of $>1,000$ charts based on the inclusion criteria. Once the inclusion and exclusion criteria were applied, a total of 96 patients were eligible for analysis.

Table I shows the overall demographics of the patients with associated P-values. There was no statistically significant difference in sex, ethnicity, smoking history, age or mean hemoglobin A1c between the control and study groups. The difference in BMI was statistically significant $(\mathrm{P}=0.0058)$, with the study cohort's mean BMI being $32.2 \mathrm{~kg} / \mathrm{m}^{2}$ and SD being $6.9 \mathrm{~kg} / \mathrm{m}^{2}$ while the control cohort had a mean BMI of $28.3 \mathrm{~kg} / \mathrm{m}^{2}$ and a SD of $6.3 \mathrm{~kg} / \mathrm{m}^{2}$. Table II shows the cancer-specific characteristics, including cancer type, stage and tumor pathologies (including adenocarcinoma, squamous, and small cell cancer) for each cohort.

Cancer specific demographics. The control group included 57 patients $(59 \%)$, out of which 30 patients $(53 \%)$ had colorectal cancer and 27 patients $(47 \%)$ had an airway cancer. Of the patients with colorectal cancer, 19 patients $(63 \%)$ had a stage 3 disease, while 11 patients $(37 \%)$ had a stage 4 disease. Among the patients in the stage 3 colorectal cancer group, all 19 patients received chemotherapy and surgical resection, and 6 patients (32\%, all cancers located in the rectum) received radiation therapy. Among the patients in the stage 4 colorectal cancer group, 9 patients $(82 \%)$ received chemotherapy, 4 patients $(36 \%)$ underwent surgical resection and 2 patients ( $18 \%$, both cancers located in the rectum) received radiation therapy. In the airway cancer control group, 17 patients (63\%) had a stage 3 disease and 10 patients $(37 \%)$ had a stage 4 disease. In the stage 3 category, 15 patients $(88 \%)$ received chemotherapy, 5 patients $(29 \%)$ underwent surgical resection and 15 patients $(88 \%)$ received concurrent radiation therapy. In the stage 4 group, 9 patients (90\%) received chemotherapy, 2 patients (20\%) received surgery (both in the head and neck subgroup) and 3 patients (30\%) received palliative radiation.

The study group included 39 patients (41\%), of those, 21 patients $(54 \%)$ had colorectal cancer and 18 patients $(46 \%)$ had an airway cancer. In the colorectal cancer study group, 18 patients $(85 \%)$ had a stage 3 disease, while only 3 patients $(14 \%)$ had a stage 4 disease. Among the patients in the stage 3 
Table I. Demographics of patients $(n=96)$.

\begin{tabular}{lcc}
\hline Characteristics & Control group $(\mathrm{n}=57 ; 59 \%)$ & Study group $(\mathrm{n}=39 ; 41 \%)$ \\
\hline Sex, $\mathrm{n}(\%)$ & & $20(51)$ \\
Male & $34(60)$ & $19(49)$ \\
Female & $23(40)$ & \\
Ethnicity, n (\%) & & $26(67)$ \\
Caucasian & $45(79)$ & $11(28)$ \\
African American & $10(18)$ & $2(5)$ \\
Other & $2(4)$ & \\
Smoking history, n $(\%)$ & & $25(64)$ \\
Yes & $41(72)$ & $14(36)$ \\
No & $16(28)$ & $66 \pm 9.3$ \\
Age, years, mean & $69 \pm 8.0$ & $32 \pm 6.9$ \\
BMI, kg/m ${ }^{2}$ mean \pm SD & $28.3 \pm 6.2$ & $7.9 \pm 1.6$ \\
Hgb Alc, $\%$ mean \pm SD & $7.2 \pm 1.5$ & 0.4190 \\
\hline
\end{tabular}

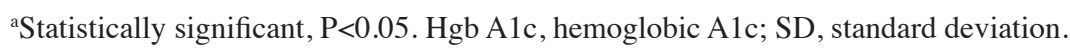

Table II. Cancer specific demographics $(n=96)$.

\begin{tabular}{lcc}
\hline Characteristic & $\begin{array}{c}\text { Control group } \\
(\mathrm{n}=57,59 \%)\end{array}$ & $\begin{array}{c}\text { Study group } \\
(\mathrm{n}=39,41 \%)\end{array}$ \\
\hline Colorectal & & \\
Stage III & $19(33)$ & $18(46)$ \\
Adeno & $19(100)$ & $18(100)$ \\
Stage IV & $11(19)$ & $3(8)$ \\
Adeno & $10(91)$ & $3(100)$ \\
Unknown & $1(9)$ & \\
Airway (Lung subgroup) & & \\
Stage III & $14(25)$ & $9(23)$ \\
Adeno & $3(21)$ & $7(77)$ \\
Squamous & $8(57)$ & $1(11)$ \\
Small & $3(21)$ & $1(11)$ \\
Stage IV & $9(16)$ & $5(13)$ \\
Adeno & $6(67)$ & $3(60)$ \\
Squamous & $1(11)$ & - \\
Small & $2(22)$ & $1(20)$ \\
Large neuroendocrine & - & $1(20)$ \\
Airway (Head/Neck subgroup) & & $3(100)$ \\
Stage III & $3(5)$ & $1(3)$ \\
Squamous & $3(100)$ & $1(100)$ \\
Stage IV & $1(2)$ & $3(8)$ \\
Squamous & & \\
\hline
\end{tabular}

colorectal cancer study group, all 18 patients underwent surgical resection and received chemotherapy, and 6 patients (33\%, all cancers located in the rectum) had radiation treatment. Among the patients in the stage 4 group, 2 patients $(67 \%)$ received chemotherapy, 2 patients $(67 \%)$ had palliative surgery and no patients received radiation. In the airway cancer study group, 10 patients $(56 \%)$ had a stage 3 disease and 8 patients
(44\%) had a stage 4 disease. Among the patients in the stage 3 group, all 10 patients received chemotherapy, 2 patients $(20 \%)$ underwent surgical resection and 8 patients $(80 \%)$ had concurrent radiation therapy. Among the patients in the stage 4 group, 7 patients $(88 \%)$ had chemotherapy, 2 patients $(25 \%)$ had neck dissection surgery and 6 patients $(75 \%)$ had radiation therapy.

With regards to location of the colorectal cancers in the study group, 9 patients had right-sided colon cancer, 6 patients had left-sided colon cancer and 5 patients had rectal cancer (the location of the carcinoma was not available for 1 patient). Three patients were tested for BRAF mutations and all were found to be wild type. In the control group, 9 patients had right-sided colon cancer, 10 patients had left-sided colon cancer and 9 patients had rectal cancer (the location was not available for 2 patients). Eight patients were tested for BRAF mutations, 6 of which were found to be wild type $(75 \%)$, 1 patient possessed a mutant phenotype and 1 patient had an indeterminate phenotype.

Progression-free survival and overall survival. With a median follow-up time of 26 months (range: 1-204 months), PFS was significantly improved in the study group as the HR was 0.42 (95\% CI: 0.21-0.84) and $\mathrm{P}=0.014$. Malignancies progressed in $23.7 \%$ of patients treated with DPP4 inhibitors, compared to $50.9 \%$ of patients in the control group; the OR was 0.303 (95\% CI: 0.106-0.809) and $\mathrm{P}=0.010$. The median antihyperglycemic treatment time of patients diagnosed with cancer was 15 months (range: 1-142 months) for the control group and 13 months (range: 1-59 months) for the study group.

We performed multivariate analyses to assess the effectiveness of the treatment by controlling for variables including cancertype,cancerstage, history of surgicalresection,non-small vs. small cell cancer (for the airway group), and right-sided vs. left-sided cancer (for the colorectal group) (Fig. 1). When controlled for cancer type (airway vs. colorectal), PFS was significantly improved in the study group with a HR of 0.42 (95\% CI: 0.21-0.83) and $\mathrm{P}=0.013$. When controlled for cancer 


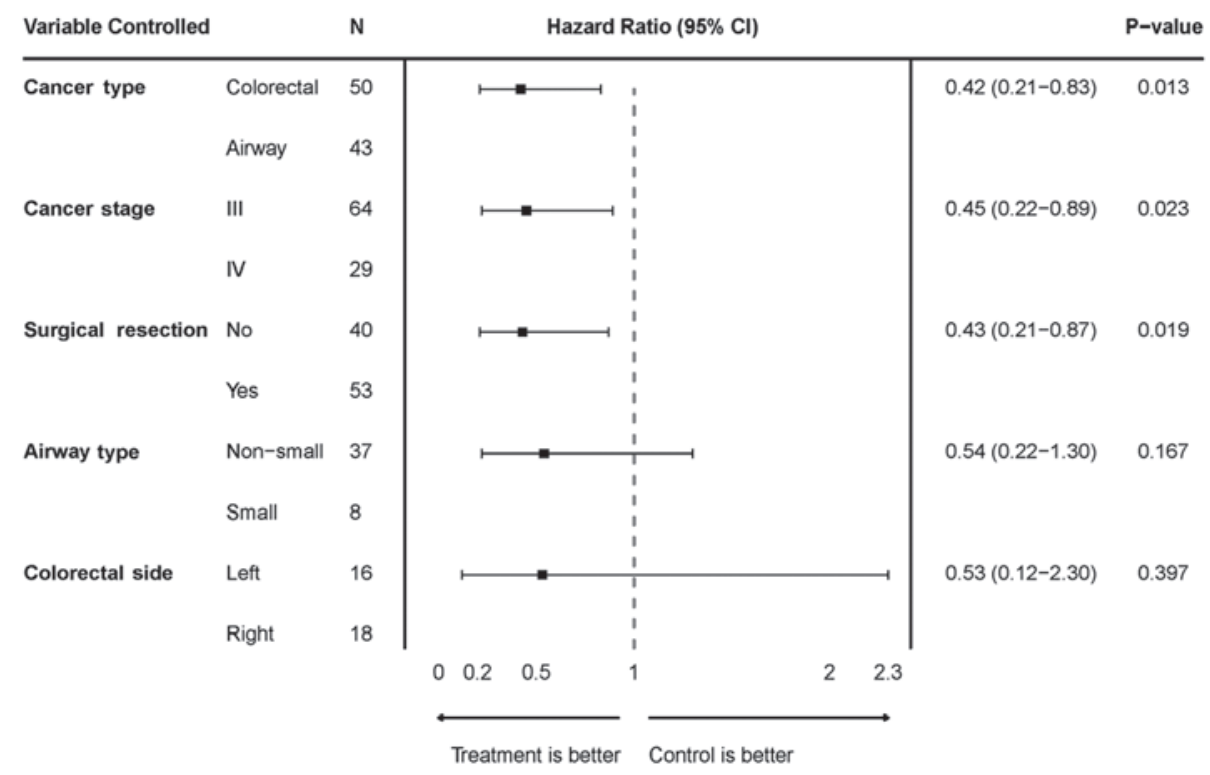

Figure 1. Hazard-ratio plot with 95\% CI comparing treatment vs. control in a multivariate analysis controlling for cancer type, cancer stage, surgical resection history, airway cancer type, and colorectal cancer laterality. CI, confidence interval.

stage (stage 3 vs. stage 4), PFS was significantly improved in the study group with a HR of 0.45 (95\% CI: $0.22-0.89)$ and $\mathrm{P}=0.023$. When controlled for surgical resection (yes vs. no), PFS was significantly improved in the study group with a HR of 0.43 (95\% CI: $0.21-0.87$ ) and $\mathrm{P}=0.019$. When controlled for non-small vs. small cell airway cancer, PFS was improved in the study group but not significantly as the HR was 0.54 (95\% CI: 0.22-1.30) and $\mathrm{P}=0.17$. When controlled for right vs. left sided colorectal cancer, PFS was improved in the study group but not significantly as the HR was 0.59 (95\% CI: 0.12-2.3) and $\mathrm{P}=0.40$. We also performed a multivariate analysis to reveal differences in PFS between the control and study groups while simultaneously controlling for cancer type, cancer stage and surgical resection. When controlling for these variables, there was a statistically significant improvement in PFS in the study group compared to control group as the HR was 0.44 (95\% CI: 0.21-0.91) and $\mathrm{P}=0.026$.

Fig. 2 shows a Kaplan-Meier curve comparing PFS between the control and study groups. The study group had a statistically significant improvement in PFS compared to the control group $(\mathrm{P}=0.01)$. Fig. 3 shows the comparison of OS between the control and study groups. Although there was trend towards improvement in OS in the study group, this difference was not statistically significant compared with the control group $(\mathrm{P}=0.11)$. The $\mathrm{OS}$ rate in control group was 71.93\% (95\% CI: 58.46-83.03) and the OS rate in the study group was $84.62 \%$ (95\% CI: 69.47-94.1\%).

\section{Discussion}

The precise role of CD26/DPP4 in tumor biology is unclear at this time. Preclinical studies have shown conflicting data with differential CD26/DPP4 expression and activity depending on the type of cancer. These studies suggest that it has a role as either a tumor suppressor or tumor activator depending on the tumor microenvironment and molecules with which CD26/DPP4 associates (1,3). However, since most of these studies involved in vitro assays, further investigations with in vivo experiments are needed to definitively establish the role of CD26/DPP4 in each cancer type.

Published studies have demonstrated that CD26/DPP4 plays a major role in the invasion and metastasis of selected cancers, and may be a novel therapeutic target $(1,2,5,6)$. There are several suggested mechanisms for cancer metastasis involving the intrinsic peptidase activity of CD26/DPP4 and its subsequent chemokine regulation, as well as its ability to bind key molecules. For example, CD26/DPP4 can upregulate the expression of matrix metalloproteinases (MMPs), which are responsible for the degradation of the extracellular matrix (ECM), a process necessary for cancer invasion and metastasis $(9,10)$. CD26/DPP4 is also associated with fibroblast activation protein (FAP)- $\alpha$, activating signal cascades that lead to a number of effects, including the formation of invadopodia-like protrusions with MMP activation (11) and suppression of anti-tumor immunity (12). CD26/DPP4 was correlated with poor survival in lung cancer patients (13). Additionally, CD26/DPP4 has been shown to bind ECM proteins, collagen and fibronectin through which it can directly participate in the process of tumor invasion $(2,14)$. CD26/DPP4 has also been shown to be a marker for hypoxia-induced factor 1 (HIF-1), which can be activated in perfusion-starved tumors and is responsible for re-initiating angiogenesis, thus leading to the development of resistance to anti-angiogenic drugs, which are otherwise now widely used in many types of cancer (15). Moreover, CD26/DPP4 may play a role in inhibiting tumor immune surveillance by cleaving CXCL10 and CXCL12, ligands that would otherwise assist in lymphocyte chemotaxis and migration $(16,17)$.

There have also been several in vivo studies with animal models demonstrating that CD26/DPP4 depletion/inhibition with either knockout mice or DPP4-inhibitors results in the tumor control of neoplasms such as melanoma (18), cervical cancer (19), colorectal cancer $(7,20,21)$ and non-small cell lung cancer (8). 


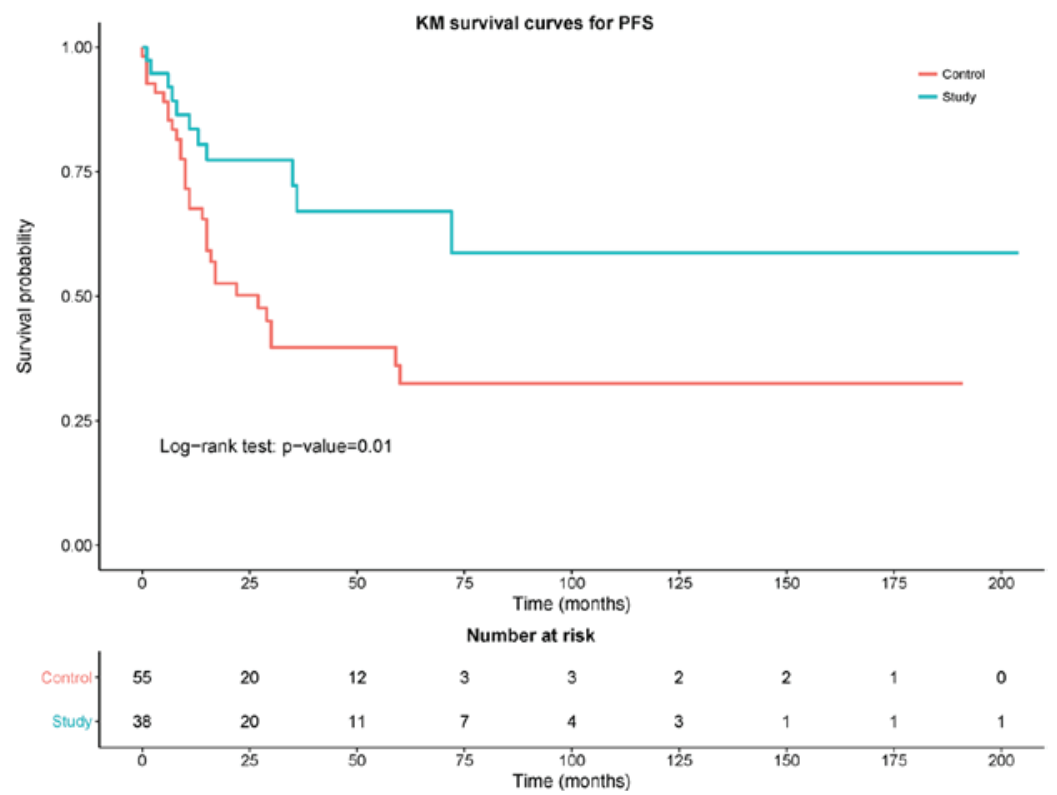

Figure 2. Kaplan-Meier curves of progression-free survival (PFS) of control (red) vs. study (blue) group with number of patients at different time points.

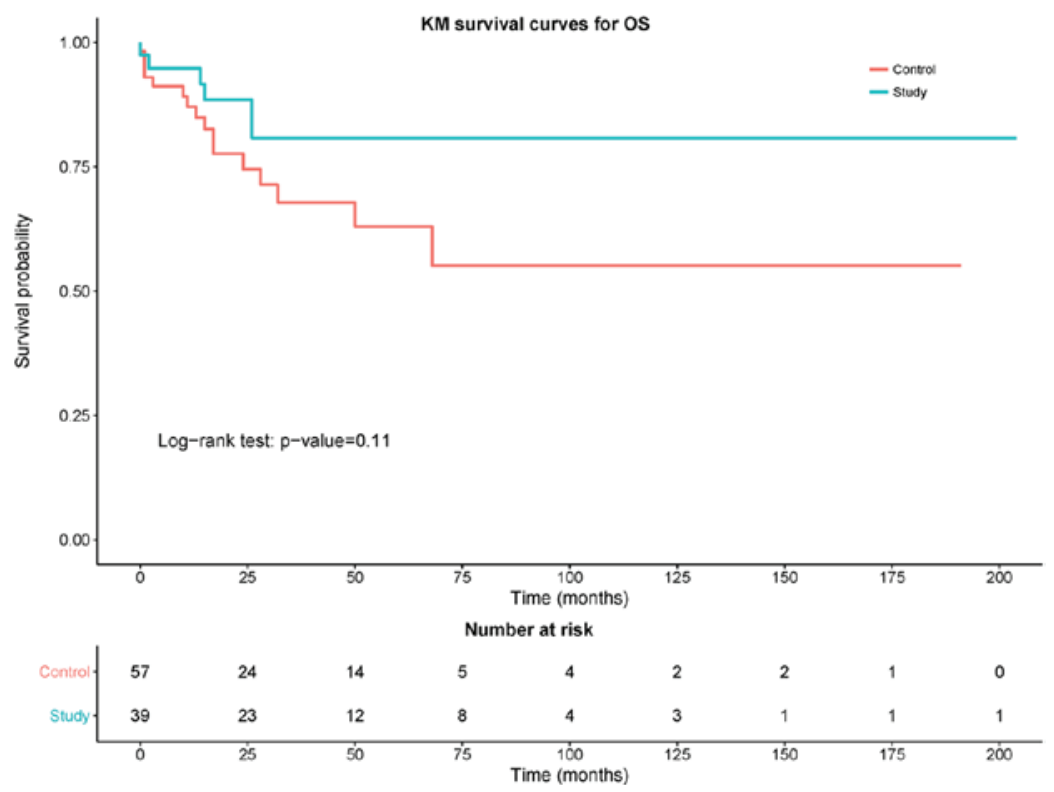

Figure 3. Kaplan-Meier curves of overall survival (OS) of control (red) vs. study (blue) group with number of patients at different time points.

To the best of our knowledge, our study is the first to evaluate the anti-tumor effect of DPP4 inhibitors in a clinical setting. We observed that the simultaneous use of DPP4 inhibitors in advanced colorectal or airway malignancies was associated with a statistically significant improvement in PFS. Of importance is the fact that DPP4-inhibitors are very well tolerated among patients with diabetes. There was a statistically significant difference when evaluated by the subgroups, including cancer types (colorectal and airway), cancer stage (stage 3 and 4) and history of surgical resection. There was a trend towards improvement in PFS in the study group, when controlled for small vs. non-small airway cancers, and right-sided vs. left-sided colorectal cancers, but the difference was not statistically significant. There was also a trend toward improvement in OS in the study group; however, the difference was not statistically significant due to the sample size being relatively small. Further subgroup analysis in OS was not performed as small sample size served as a limitation to accurately analyze further results with statistical value.

We recognize that there are limitations to the data and analysis inherent to the study design as a retrospective review. For instance, there was a potential sampling bias as the size of the stage 3 colorectal cancer cohort was larger compared to the stage 4 colorectal cancer cohort; in the study group, $86 \%$ of patients had stage 3 cancer vs. $14 \%$ of patients with stage 4 cancer and in the control group, $63 \%$ of patients had stage 3 cancer vs. $37 \%$ of patients with stage 4 cancer. While the disproportion in colorectal cancers can certainly bias the results to benefit the study group, we did notice that the control group also had more stage 3 patients. When controlled 
for stage 3 vs. stage 4, PFS was significantly improved in the study group. Moreover, when cancer type, stage and resection history were all controlled, PFS was significantly improved in the study group, further strengthening our results. Mean BMI was also statistically significant $\left(32 \mathrm{~kg} / \mathrm{m}^{2}\right.$ in the study group vs. $28.3 \mathrm{~kg} / \mathrm{m}^{2}$ in the control group). The effect of the difference in mean BMI is less clear; nonetheless, the obesity paradox has to be considered as a potential bias to benefit the study group. We also recognize that while lung, and head and neck cancers share several features and risk factors, they do not have an identical pathophysiology or even treatment approach. As stage 3 and 4 malignancies were chosen to minimize follow up times, this also serves as a potential limitation that early stage malignancies were excluded in favor of the study design. Nonetheless, even with these biases and limitations, the degree of improvement in PFS is noteworthy and statistically significant. A strength of our study is our multi-center approach, which helps to reduce the bias inherent to treatment patterns and other confounding factors present at a single institution. Further investigations with a larger retrospective database, such as the SEER database, and randomized, prospective trials that include multiple cancer types and all stages are warranted as the next step. As previously mentioned, the OS data was not statistically significant in this study, we hope to investigate this further in our large retrospective database to study the relationship between DPP4 inhibitors and malignancies, and their effect on OS. We will be analyzing data from a larger database to validate these results.

\section{Acknowledgements}

The authors would like to thank the UF Health Integrated Data Repository and the Ochsner Health System Electronic Research Submission System for providing us with the data. This abstract was previously accepted at 2018 American Society of Clinical Oncology on June 1-5th in Chicago, Illinois as an online-only publication (abstract ID no. 213185).

\section{Funding}

No funding was received.

\section{Availability of data and materials}

Data was obtained from hospital charts. All data generated or analyzed during this study are included in this published article.

\section{Authors' contributions}

AA, AF and WPS made contributions to the design of the study, IRB approval, data collection, data interpretation, drafting and critically revising the manuscript, and gave final approval of the version to be published.

YW and SM contributed to the design of the study, data analysis, data interpretation, drafting of the manuscript, and gave final approval of the version to be published.

CS and RB made contributions in drafting and critically revising the manuscript, data interpretation, and gave final approval of the version to be published.
NHD and LHD contributed to the overall study design, IRB approval, data collection, data interpretation, drafting and critically revising the manuscript, and gave final approval of the version to be published.

\section{Ethics approval and consent to participate}

The study was approved by UF IRB. The study was approved by University of Florida Institutional Review Board (IRB no. IRB201500588); and Louisiana State University Health Science Center, New Orleans, Human Research Protection Program and Institutional Review Board (IRB no.IRB00000177) and included a waiver of individual patient consent.

\section{Patient consent for publication}

Not applicable.

\section{Competing interests}

The authors declare that they have no competing interests.

\section{References}

1. Havre PA, Abe M, Urasaki Y, Ohnuma K, Morimoto C and Dang NH: The role of CD26/dipeptidyl peptidase IV in cancer. Front Biosci 13: 1634-1645, 2008.

2. Pro B and Dang NH: CD26/dipeptidyl peptidase IV and its role in cancer. Histol Histopathol 19: 1345-1351, 2004.

3. Beckenkamp A, Davies S, Willig JB and Buffon A: DPPIV/CD26: A tumor suppressor or a marker of malignancy? Tumour Biol 37: 7059-7073, 2016.

4. Javidroozi M, Zucker S and Chen WT: Plasma seprase and DPP4 levels as markers of disease and prognosis in cancer. Dis Markers 32: 309-320, 2012.

5. Angevin E, Isambert N, Trillet-Lenoir V, You B, Alexandre J, Zalcman G, Vielh P, Farace F, Valleix F, Podoll T, et al: First-in-human phase 1 of YS110, a monoclonal antibody directed against CD26 in advanced CD26-expressing cancers. Br J Cancer 116: 1126-1134, 2017.

6. Ho L, Aytac U, Stephens LC, Ohnuma K, Mills GB, McKee KS Neumann C, LaPushin R, Cabanillas F, Abbruzzese JL, et al: In vitro and in vivo antitumor effect of the anti-CD26 monoclonal antibody 1F7 on human CD30+ anaplastic large cell T-cell lymphoma Karpas 299. Clin Cancer Res 7: 2031-2040, 2001.

7. Femia AP, Raimondi L, Maglieri G, Lodovici M, Mannucci E and Caderni G: Long-term treatment with Sitagliptin, a dipeptidyl peptidase-4 inhibitor, reduces colon carcinogenesis and reactive oxygen species in 1,2-dimethylhydrazine-induced rats. Int J Cancer 133: 2498-2503, 2013.

8. Tang Z, Li J, Shen Q, Feng J, Liu H, Wang W, Xu L, Shi G, Ye X, Ge M, et al: Contribution of upregulated dipeptidyl peptidase 9 (DPP9) in promoting tumoregenicity, metastasis and the prediction of poor prognosis in non-small cell lung cancer (NSCLC). Int J Cancer 140: 1620-1632, 2017.

9. Gonzalez-Gronow M, Grenett HE, Weber MR, Gawdi G and Pizzo SV: Interaction of plasminogen with dipeptidyl peptidase IV initiates a signal transduction mechanism which regulates expression of matrix metalloproteinase-9 by prostate cancer cells. Biochem J 355: 397-407, 2001.

10. Havre PA, Dang LH, Ohnuma K, Iwata S, Morimoto C and Dang NH: CD26 expression on T-anaplastic large cell lymphoma (ALCL) line Karpas 299 is associated with increased expression of versican and MT1-MMP and enhanced adhesion. BMC Cancer 13: 517, 2013.

11. Ghersi G,Zhao Q, Salamone M, Yeh Y,Zucker S and Chen WT: The protease complex consisting of dipeptidyl peptidase IV and seprase plays a role in the migration and invasion of human endothelial cells in collagenous matrices. Cancer Res 66: 4652-4661, 2006.

12. Kraman M, Bambrough PJ, Arnold JN, Roberts EW, Magiera L, Jones JO, Gopinathan A, Tuveson DA and FearonDT: Suppression of antitumor immunity by stromal cells expressing fibroblast activation protein-alpha. Science 330: 827-830, 2010. 
13. Liao Y, Ni Y, He R, Liu W and Du J: Clinical implications of fibroblast activation protein- $\alpha$ in non-small cell lung cancer after curative resection: A new predictor for prognosis. J Cancer Res Clin Oncol 139: 1523-1528, 2013.

14. Dang NH, Torimoto Y, Schlossman SF and Morimoto C: Human CD4 helper T cell activation: Functional involvement of two distinct collagen receptors, 1F7 and VLA integrin family. J Exp Med 172: 649-652, 1990.

15. Dang DT, Chun SY, Burkitt K, Abe M, Chen S, Havre P, Mabjeesh NJ, Heath EI, Vogelzang NJ, Cruz-Correa M, et al: Hypoxia-inducible factor-1 target genes as indicators of tumor vessel response to vascular endothelial growth factor inhibition. Cancer Res 68: 1872-1880, 2008.

16. Ohnuma K, Hatano R and Morimoto C: DPP4 in anti-tumor immunity: Going beyond the enzyme. Nat Immunol 16: 791-792, 2015.

17. Janssens R, Mortier A, Boff D, Ruytinx P, Gouwy M, Vantilt B, Larsen O, Daugvilaite V, Rosenkilde MM, Parmentier M, et al: Truncation of CXCL12 by CD26 reduces its CXC chemokine receptor 4- and atypical chemokine receptor 3-dependent activity on endothelial cells and lymphocytes. Biochem Pharmacol 132: 92-101, 2017.
18. Barreira da Silva R, Laird ME, Yatim N, Fiette L, Ingersoll MA and Albert ML: Dipeptidylpeptidase 4 inhibition enhances lymphocyte trafficking, improving both naturally occurring tumor immunity and immunotherapy. Nat Immunol 16: 850-858, 2015.

19. Beckenkamp A, Willig JB, Santana DB, Nascimento J, Paccez JD, Zerbini LF, Bruno AN, Pilger DA, Wink MR and Buffon A: Differential expression and enzymatic activity of DPPIV/CD26 affects migration ability of cervical carcinoma cells. PLoS One 10: e0134305, 2015.

20. Jang JH, Baerts L, Waumans Y, De Meester I, Yamada Y,Limani P, Gil-Bazo I, Weder W and Jungraithmayr W: Suppression of lung metastases by the CD26/DPP4 inhibitor Vildagliptin in mice. Clin Exp Metastasis 32: 677-687, 2015.

21. Lam CS, Cheung AH, Wong SK, Wan TM, Ng L, Chow AK, Cheng NS, Pak RC, Li HS, Man JH, et al: Prognostic significance of CD26 in patients with colorectal cancer. PLoS One 9: e98582, 2014. 